\title{
Излучательные характеристики мощных полупроводниковых лазеров (1060 нм) с узким мезаполосковым контактом на основе асимметричных гетероструктур AIGaAs/GaAs c широким волноводом
}

\author{
(C) И.С. Шашкин, А.Ю. Лешко, Д.Н. Николаев, В.В. Шамахов, Н.А. Рудова, К.В. Бахвалов, А.В. Лютецкий, \\ В.А. Капитонов, В.В. Золотарев, С.О. Слипченко, Н.А. Пихтин, П.С. Копьев
}

Физико-технический институт им. А.Ф. Иоффе Российской академии наук, 194021 Санкт-Петербург, Россия

E-mail: shashkin@mail.ioffe.ru

Поступила в Редакцию 12 декабря 2019 г.

В окончательной редакции 23 декабря 2019 г.

Принята к публикации 23 декабря 2019 г.

\begin{abstract}
Исследованы излучательные характеристики лазеров с узким мезаполосковым контактом (ширина 5.5 мкм) на основе асимметричных $\mathrm{AlGaAs} / \mathrm{GaAs}$ гетероструктур. Показано, что максимальная мощность в непрерывном режиме ограничена тепловым разогревом и достигает 1695 мВт на токе 2350 мА при $25^{\circ} \mathrm{C}$, а максимальный кпд достигает 54.8\%. Понижение рабочей температуры до $-8^{\circ} \mathrm{C}$ позволило повысить максимальную непрерывную мощность в маломодовом режиме до 2 Вт. При накачке импульсами тока длительностью 240 нс и амплитудой 4230 мА была получена пиковая мощность 2930 мВт. Показано, что в импульсном режиме существует область „оптического провала“, в которой наблюдается низкоэффективная лазерная генерация с режимом генерации периодической последовательности лазерных импульсов суб-нс длительности.
\end{abstract}

Ключевые слова: одномодовый лазер, $\mathrm{AlGaAs} / \mathrm{GaAs}$, оптический провал, моды высшего порядка, пиковая мощность.

DOI: 10.21883/FTP.2020.04.49149.9333

\section{1. Введение}

Традиционно мощные полупроводниковые лазеры рассматриваются как эффективные источники накачки активных сред волоконных и твердотельных лазерных систем для решения множества задач, в том числе связанных с обработкой материалов. Однако в последние годы активно развивается направление, в котором мощные лазерные системы используют энергию только полупроводниковых лазеров (так называемое направление Direct Diode Laser Application) [1]. Ключевая задача, которую необходимо решить при создании таких систем, связана с созданием излучателей, обладающих высоким качеством лазерного излучения и одновременно высокой выходной оптической мощностью. Одно из решений основано на создании мощных одномодовых и маломодовых полупроводниковых лазеров. В последние годы наиболее высокие результаты были продемонстрированы одномодовыми полупроводниковыми лазерами на основе гетероструктур со сверхшироким волноводом. Так, в работе [2] базовая конструкция была основана на гетероструктуре с асимметричными эмиттерами и градиентным волноводным слоем толщиной 3.4 мкм, в результате была продемонстрирована непрерывная мощность в одномодовом режиме 1 Вт для полоска шириной 10 мкм и в маломодовом режиме 2.7 Вт для полоска шириной 15 мкм. В работе [3] были исследованы гетероструктуры на основе одномерных фотонных кристаллов с волноводом толщиной 7 мкм, что позволило получить выходную оптическую мощность 2 Вт в одномодовом режиме для полоска шириной 7 мкм. В рамках данной работы исследованы возможности асимметричных гетероструктур с расширенным волноводом для решения задачи генерации мощного лазерного излучения при использовании конструкции кристаллов с узким мезаполосковым контактом.

\section{2. Экспериментальные образцы}

В качестве базовой была выбрана асимметричная гетероструктура, позволяющая получать низкие внутренние оптические потери и, таким образом, решать задачу увеличения длины резонатора без существенного падения эффективности, что позволяет эффективно отводить тепло от кристаллов мощных полупроводниковых лазеров. Гетероструктура включала широкозонные $n$ и $p$-эмиттеры на основе $\mathrm{Al}_{x} \mathrm{Ga}_{1-x} \mathrm{As}(x=0.27)$ толщиной 1.5 мкм каждый, волноводный слой на основе $\mathrm{Al}_{x} \mathrm{Ga}_{1-x} \mathrm{As} \quad(x=0.1)$ толщиной 1.7 мкм и активную область InGaAs толщиной 9 нм, смещенную на 0.2 мкм относительно центра волновода в сторону $p$-эмиттера. Предварительная характеризация рассматриваемой гетероструктуры, проведенная с использованием полупроводниковых лазеров с широким полосковым контактом, показала, что пороговая плотность тока при бесконечной длине резонатора составила $60 \mathrm{~A} / \mathrm{cm}^{2}$, а внутренние оптические потери $0.6 \mathrm{~cm}^{-1}$, при внутреннем квантовом выходе, близком к $100 \%$. 


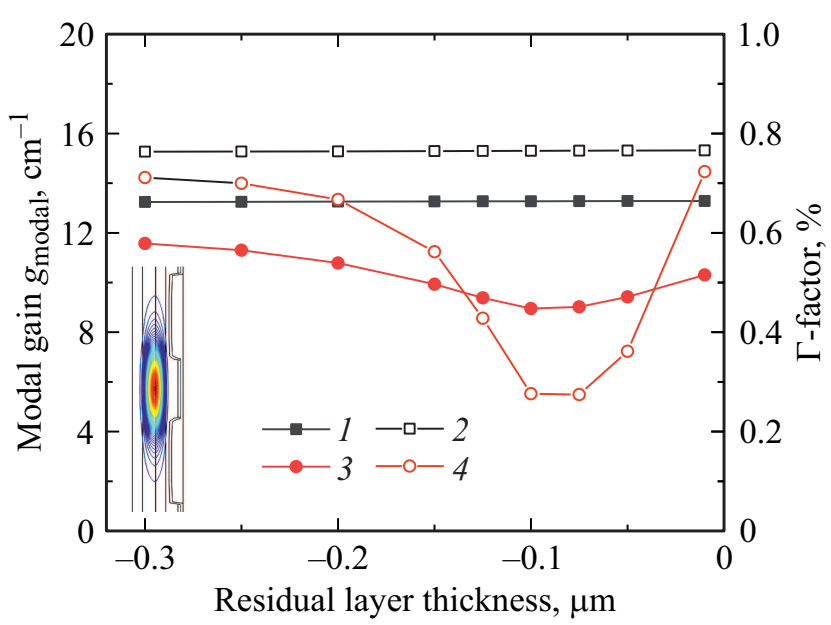

Рис. 1. Расчетная зависимость модального усиления и фактора оптического ограничения мод $\mathrm{TE}_{00}(1,2)$ и $\mathrm{TE}_{10}(3,4)$ для 2D-волновода при различных значениях параметра RLT (residual layer thickness) для ширины мезаполоска 5.5 мкм. На вставке - профиль ТЕ 00 моды 2D-волновода при $\mathrm{RTL}=-0.29$ мкм.

В рамках данной работы для реализации одномодового режима генерации использовалась конструкция „мелкая меза“, в которой латеральный волновод формируется двумя мезаканавками, а его характеристики определяются шириной мезаполоскового контакта и глубиной травления. На первом этапе был проведен расчет параметров конструкции для ширины мезаполоскового контакта 5.5 мкм. В проведенном расчете определялась не только форма моды и фактора оптического ограничения в активной области, но также проводился расчет модального усиления нулевой моды и мод более высокого порядка с учетом растекания тока и оптических потерь. Именно модальное усиление характеризует возможность модовой селекции и работы лазера в одномодовом режиме. Более высокое значение модального усиления одной из мод дает преимущество в выполнении пороговых условий генерации, в результате порог для данной моды будет достигнут в первую очередь. Расчетные зависимости модального усиления и фактора оптического ограничения для мод $\mathrm{TE}_{00}$ и $\mathrm{TE}_{01}$ от остаточной толщины $p$-эмиттера (RLT - Residual layer thickness; $\mathrm{RLT}=W-D$, где $W-$ глубина мезаканавки, $D-$ суммарная толщина р-эмиттера и контактного слоя) показаны на рис. 1. Видно, что в достаточно широком диапазоне значений RLT наблюдается преимущество модального усиления моды $\mathrm{TE}_{00}$ над модой $\mathrm{TE}_{01}$.

На основе проведенных расчетов были изготовлены одномодовые лазеры со значением RLT $=-0.29$ мкм, шириной мезаполоска 5.5 мкм и длиной резонатора 3 мм, на грани были нанесены просвеляющие (5\%) и отражающие (95\%) покрытия. Просветляющие покрытия формировались на основе пленок AlN [4]. Изготовленные кристаллы были напаяны полоском вниз на медные теплоотводы с помощью индиевого припоя.

\section{3. Экспериментальные результаты}

В рамках экспериментальных исследований на первом этапе были проведены измерения ватт-амперных характеристик (ВтАХ) в непрерывном режиме генерации. На рис. 2 показаны зависимости непрерывной оптической мощности и энергетической эффективности для рабочих температур $+25,+10$ и $-8^{\circ} \mathrm{C}$. Видно, что основным фактором, ограничивающим максимальную выходную мощность в непрерывном режиме, является тепловой разогрев лазерного кристалла. Так, максимальная мощность при $25^{\circ} \mathrm{C}$ достигала $1695 \mathrm{mB}$ на токе 2350 мА, при этом максимальный кпд составил 54.8\% на токе 550 мА. Снижение рабочей температуры за счет системы термостабилизации позволяет сместить точку термического насыщения в область больших токов. В результате при температуре $-8^{\circ} \mathrm{C}$ максимальная мощность достигала 2 Вт на токе $2650 \mathrm{MA}$, при этом максимальный кпд составил 57.8\% на токе 300 мА. Другой важной характеристикой является расходимость излучения. Экспериментальные и расчетные дальние поля в сечениях, перпендикулярных и параллельных $p-n$-переходу, показаны на рис. 3. Расходимость в плоскости, перпендикулярной слоям гетероструктуры, составила $29.4^{\circ}$ на уровне половина от максимума интенсивности и не менялась с током накачки (рис. $3, a$ ). Расходимость излучения в параллельной плоскости для начального участка ВТАX сохраняла симметричную форму, близкую к расчетному профилю нулевой моды, с незначительными искажениями в виде низкоинтенсивных „ушей“ (рис. 3, $b$, ток 0.15 А). По мере приближения к области „кинка“ на ВТАХ форма поля деформиро-

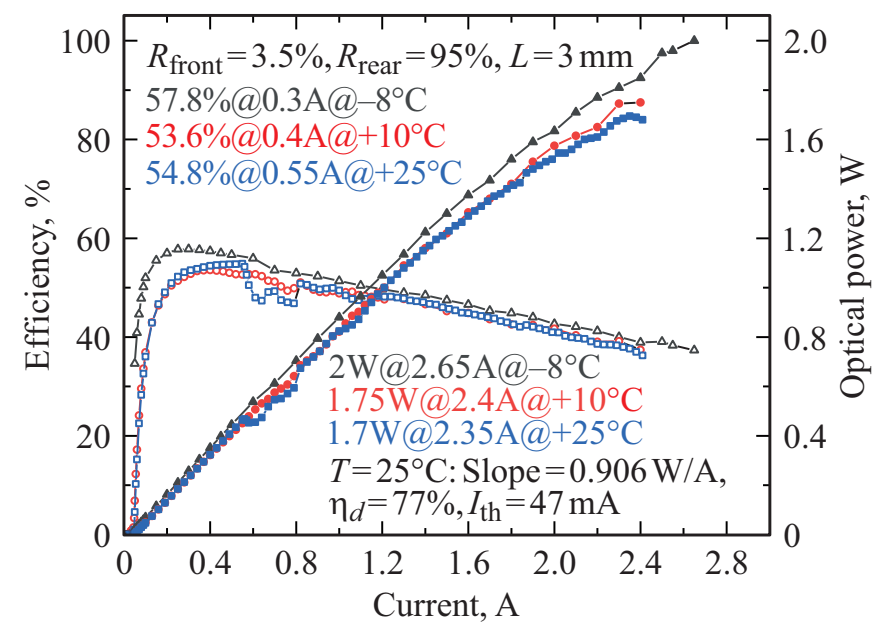

Рис. 2. Зависимости кпд (открытые символы) и выходной оптической мощности (закрашенные символы) в непрерывном режиме генерации для полупроводниковых лазеров конструкции мелкая меза с нанесенными на грани резонатора просветляющими (3.5\%) и отражающими $(95 \%)$ покрытиями: квадраты - температура теплоотвода $+25^{\circ} \mathrm{C}$, кружки - температура теплоотвода $+10^{\circ} \mathrm{C}$, треугольники - температура теплоотвода $-8^{\circ} \mathrm{C}$. Длина резонатора 3 мм, ширина мезаполоска 5.5 мкм. 

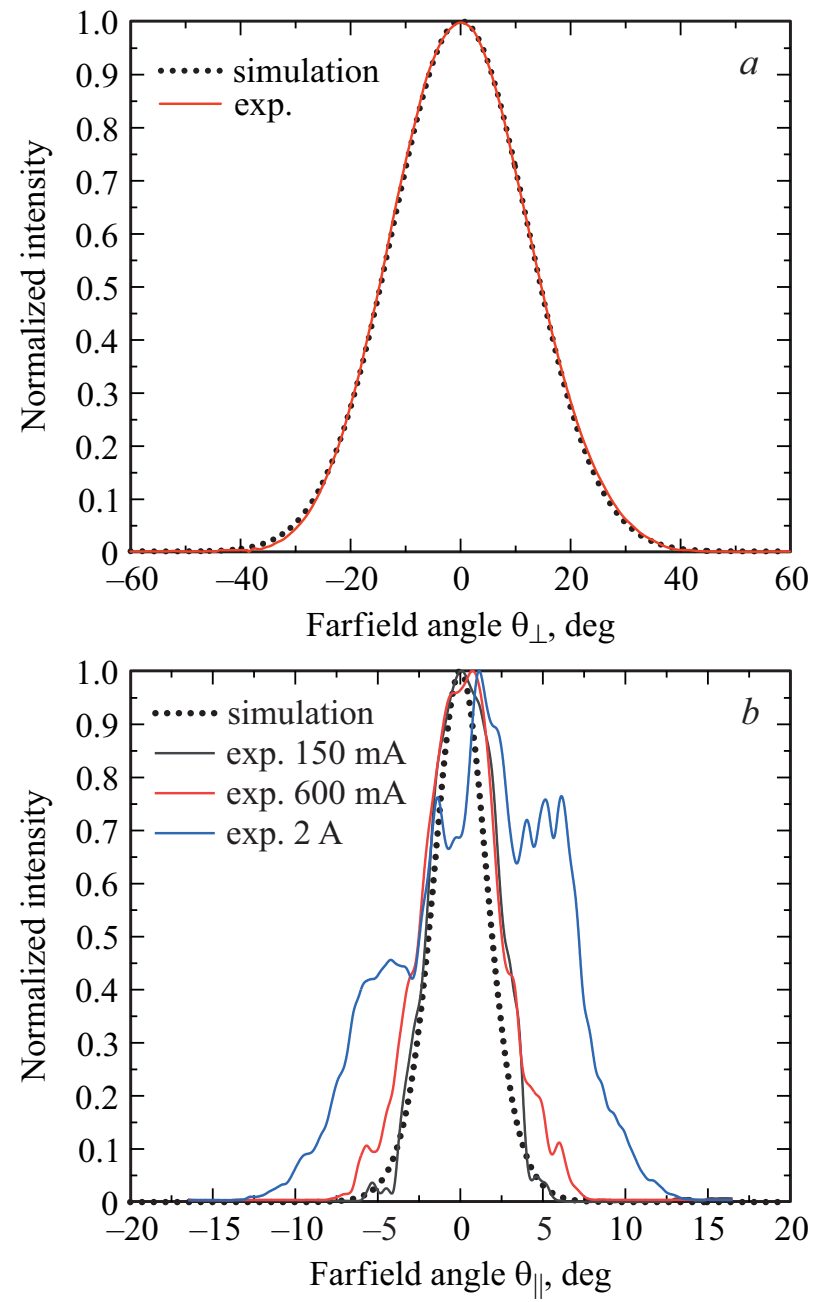

Рис. 3. Распределение излучения в дальней зоне для полупроводниковых лазеров конструкции мелкая меза, работающих в непрерывном режиме генерации, в плоскости, перпендикулярной $(a)$ и параллельной $(b) p-n$-переходу. Непрерывные линии - эксперимент, пунктирная линия - расчет. Длина резонатора 3 мм, ширина мезаполоска 5.5 мкм, температура теплоотвода $25^{\circ} \mathrm{C}$.

валась за счет уширения нижней части распределения (рис. $3, b$, ток $0.6 \mathrm{~A}$ ). В области максимальных значений токов поле имело несимметричную структуру с несколькими пиками, характерную для генерации в маломодовом режиме (рис. $3, b$, ток 2 А). Расчет расходимости поля в параллельной плоскости продемонстрировал достаточно хорошее совпадение с экспериментом при низких токах, что свидетельствует об адекватности используемой модели расчета 2D-волновода.

Для оценки предельных возможностей разработанных полупроводниковых лазеров с узким мезаполосковым контактом были исследованы ВтАХ при накачке импульсами длительностью 240 нс. Использование импульсной накачки позволило существенно снизить тепловой разогрев и, таким образом, повысить уровень тока накачки. Измерения динамики лазерной генерации проводились с помощью InGaAs фотодетектора и осциллографа с полосой частот 6 ГГц. На рис. 4 показана динамика лазерной генерации. Видно, что до тока 1 А форма оптического импульса с достаточной степенью точности повторяет форму импульса тока. Дальнейшее увеличение тока сопровождается возникновением так называемого „оптического провала“ в начальной части лазерного импульса. При этом наблюдаемые интенсивные пики

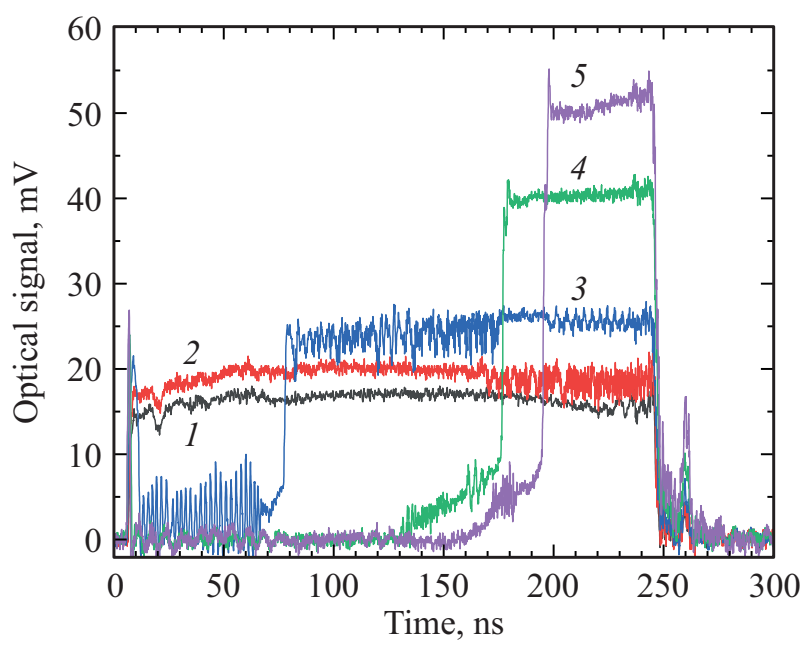

Рис. 4. Зависимость спектрально интегрированной оптической мощности от времени для полупроводникового лазера с длиной резонатора 3 мм и нанесенными просветляющими (3.5\%) и отражающими (95\%) покрытиями. Значение амплитуды импульса тока накачки, А: $1-1,2-1.2,3-1.6,4-2.8$, 5 - 4. Длин резонатора 3 мм, ширина мезаполоска 5.5 мкм, температура теплоотвода $25^{\circ} \mathrm{C}$.

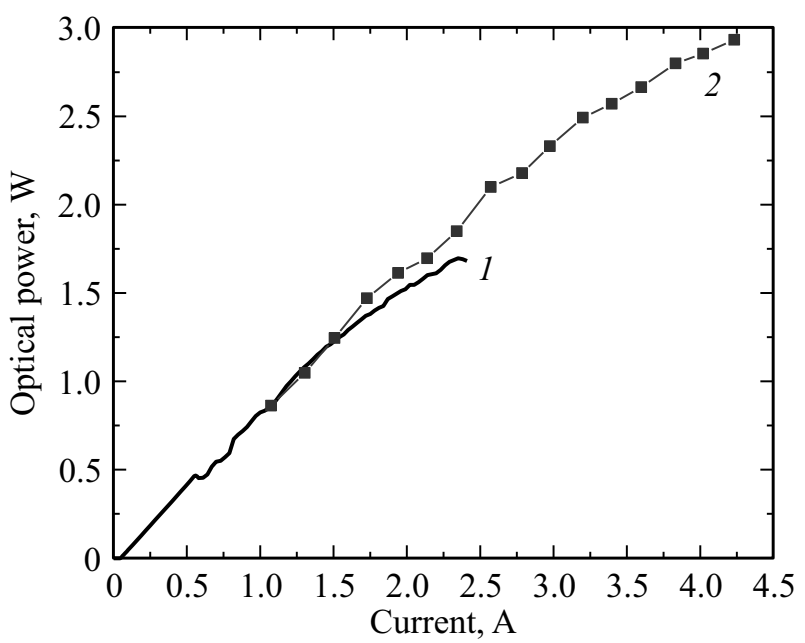

Рис. 5. Зависимость выходной оптической мощности в непрерывном (1) и импульсном (2) режимах генерации для полупроводникового лазера конструкции мелкая меза с длиной резонатора 3 мм и нанесенными просветляющими (3.5\%) и отражающими $(95 \%)$ покрытиями. Пиковая мощность в импульсном режиме взята для момента времени $222 \mathrm{Hc}$ от начала импульса. Длина резонатора 3 мм, ширина мезаполоска 5.5 мкм, температура теплоотвода $25^{\circ} \mathrm{C}$. 
лазерной генерации при включении импульса тока могут быть обусловлены недостаточной резкостью фронта включения тока, который составляет 3 нс. Увеличение амплитуды импульса тока сопровождается расширением области „оптического провала“. На рис. 5 показана зависимость пиковой мощности от амплитуды тока накачки, соответствующей 222 нс от начала импульса. Видно, что переход к импульсному режиму позволил расширить диапазон токов, в результате продемонстрирована пиковая мощность 2930 мВт при амплитуде импульсов тока 4230 мА. Видно, что даже при импульсной накачке имеет место отклонение ВТАХ от линейности. Необходимо отметить, что в данном случае максимальные плотности тока и уровни мощности, достигаемые в полупроводниковых лазерах с узким мезаполосковым контактом, сопоставимы с характерными значениями, при которых наблюдается нетепловое насыщение ВтАХ в мощных многомодовых лазерах $[5,6]$.

Рассмотрим подробнее особенности лазерной динамики для токов, при которых появляется „оптический провал“ (в качестве такого значения тока был выбран ток 1.6 А). Для этого проводилась спектральная селекция излучения лазера с узким мезаполосковым контактом. В используемой схеме лазерный луч пропускался через монохроматор, на выходе которого располагался быстрый фотоприемник, подключенный к осциллографу. Предложенная схема позволяла исследовать динамику лазерной генерации на выбранной длине волны, в полосе, соответствующей спектральному разрешению схемы монохроматора. Первая особенность связана с тем, что в процесс лазерной генерации вовлечен достаточно широкий диапазон длин волн. Видно, что наблюдаемые сигналы в области „оптического провала“ с различной интенсивностью захватывали спектральный диапазон 1046-1062 нм (рис. 6). При этом коротковолновая и длинноволновая части спектров высвечивались только в области „оптического провала“ (рис. 6, спектральные диапазоны 1046-1054 и 1060-1062 нм), а при переходе к эффективной лазерной генерации излучение в этих частях спектра выключалось. Тогда как центральная часть спектра имела низкоинтенсивный сигнал как в области „оптического провала“, так и во время высокоинтенсивной лазерной генерации. Видно, что в области „оптического провала“ лазерная генерация имеет характер периодической последовательности импульсов длительностью не более 270 пс (возможно, что приведенное значение ограничено разрешающей способностью приборов, а реальная длительность лазерных импульсов еще меньше), при этом частота повторения непрерывно изменялась от $\sim 470$ МГц в начале области до $\sim 1$ ГГц в конце области „оптического провала“. Также видно, что высокоэффективная генерация происходит только в центральной части наблюдаемого спектра 1057-1059 нм (рис. 6, спектральный диапазон 1057-1059 нм), при этом в данной области наблюдаются процессы конкуренции модовых структур, так как лазерная динамика, измеренная со спектральным разрешением, имеет явную

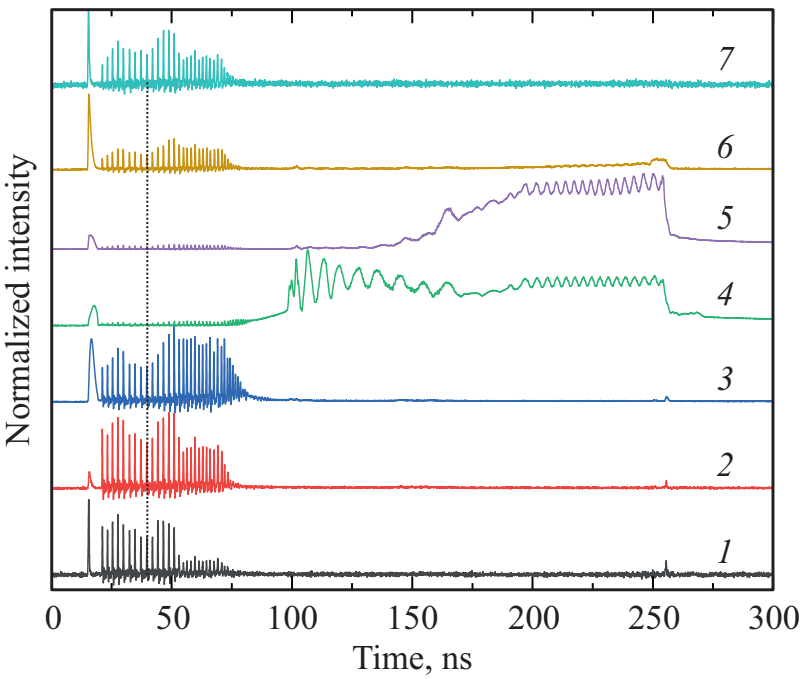

Рис. 6. Зависимость спектрально селектированной оптической мощности от времени для полупроводникового лазера с длиной резонатора 3 мм и нанесенными просветляющими $(3.5 \%)$ и отражающими (95\%) покрытиями, полученная для амплитуды импульса тока накачки 1.6 А на длине волны, нм: $1-1046$, $2-1050,3-1054,4-1057,5-1059,6-1060,7-1062$. Температура теплоотвода $25^{\circ} \mathrm{C}$.

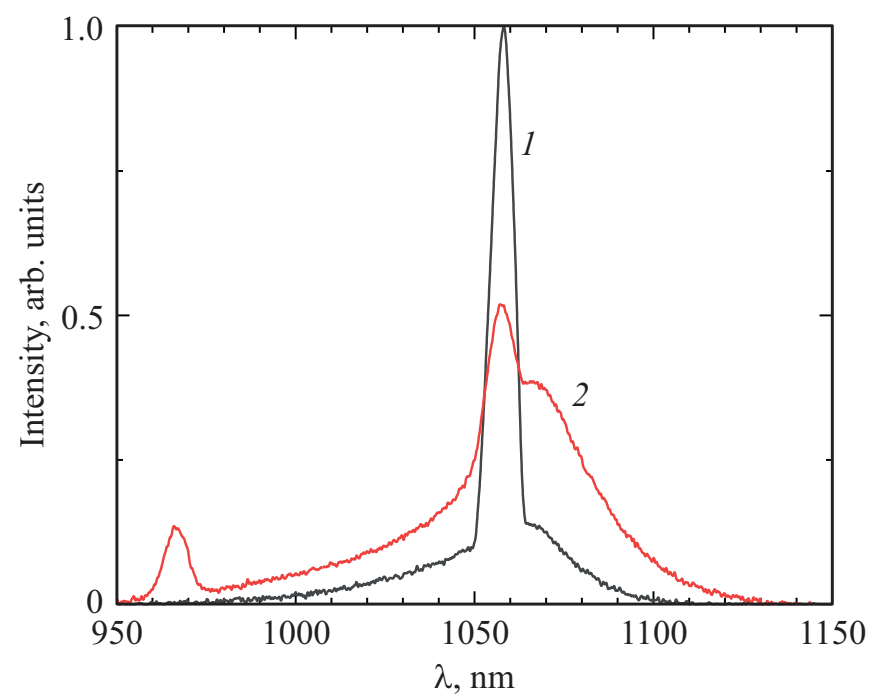

Рис. 7. Спектры спонтанного излучения полупроводникового лазера с длиной резонатора 3 мм и нанесенными просветляющими $(3.5 \%)$ и отражающими $(95 \%)$ покрытиями, полученные при накачке импульсами длительностью 78 нс и амплитудой $1 \mathrm{~A}(1)$ и $1.6 \mathrm{~A}(2)$. Температура теплоотвода $25^{\circ} \mathrm{C}$.

высокочастотную модуляцию (рис. 6, спектральный диапазон 1057-1059 нм). Возможность проведения вертикальной линии (рис. 6, пунктирная линия) для одного момента времени для разных длин волн через пики в зависимостях доказывает, что имеют место именно биения синхронизованных латеральных мод [7]. Область „оптического провала“ наблюдалась ранее в работах, посвященных исследованиям нетепловых эффектов па- 
дение излучательной эффективности многомодовых и одномодовых полупроводниковых лазеров [8-10]. В качестве основных факторов отмечалось включение высокодобротных мод в многомодовых лазерах $[8,9]$ и коллапс латерального волновода в одномодовых лазерах [10]. В нашем случае основная отличительная черта связана с присутствием низкоэффектиной генерации в области „оптического провала“. Чтобы определиться, какой из указанных факторов выполняет определяющую роль, были проведены исследования спектров спонтанного излучения при длительности импульса 78 нс и токах накачки $1 \mathrm{~A}$, когда область „оптического провала“ отсутствует, и $1.6 \mathrm{~A}$, когда лазерная динамика включает область „оптического провала“. Из полученных зависимостей видно (рис. 7) что появление области оптического провала сопровождается существенным уширением спектра лазерной генерации, о чем явно свидетельствует появившийся дополнительный высокоэнергетический пик в области 967 нм, соответствующий переходу $2 e-2 h h$. Это показывает, что концентрация в активной области при работе в области „оптического провала“ заметно возрастает, что не соответствует наблюдениям работ $[8,9]$, в которых включение высокодобротных мод должно сопровождаться незначительными изменениями пороговых условий и снижением пороговой концентрации. При этом коллапс латерального волновода, при котором наблюдаются заметное падение фактора оптического ограничения моды в активной области и существенное повышение порога генерации, может быть одной из причин наблюдаемого падения излучательной эффективности в области „оптического провала“. При этом восстановление волноводных свойств возможно, в том числе, и за счет тепловых эффектов, связанных с локальным разогревом в области полоскового контакта.

\section{4. Заключение}

В работе представлены результаты исследований излучательных характеристик мощных полупроводниковых лазеров с узким мезаполосковым контактом. Показано, что в зависимости от уровня возбуждения и режима накачки различные факторы определяют максимальную оптическую мощность и модовую структуру лазерного излучения. Показано, что использование асимметричных структур с низкими оптическими потерями позволяет сохранить излучательную эффективность для кристаллов с достаточно длинными резонаторами, что важно для работы в непрерывном режиме генерации, при этом повышение температурной стабильности излучательных характеристик может позволить в будущем повысить максимальное значение мощности, которое в проведенных экспериментах было ограничено тепловым разогревом. Исследования в импульсном режиме показали, что сформированный латеральный волновод имеет достаточно высокую чувствительность к таким факторам, как накопление носителей заряда и тепловой разогрев, поэтому поиск решений, связанных со стабилизацией волноводной структуры при высоких уровнях возбуждения, будет одним из основных требований при разработке лазеров, работающих при сверхвысоких уровнях токовой накачки импульсами длительностью единицы и десятки нс.

\section{Финансирование работы}

Исследование выполнено за счет гранта Российского научного фонда (проект № 19-79-30072)

\section{Конфликт интересов}

Авторы заявляют, что у них нет конфликта интересов.

\section{Список литературы}

[1] G.C. Rodrigues, H. Vanhove, J.R. Duflou. Phys. Procedia, 56, 901 (2014).

[2] M. Wilkens, H. Wenzel, J. Fricke, A. Maabdorf, P. Ressel, S. Strohmaier, A. Knigge, G. Erbert, G. Trankle. IEEE Photon. Technol. Lett., 30 (6), 545 (2018).

[3] S. Zhao, H. Qu, Y Liu, L. Li, Y. Chen, X. Zhou, Y. Lin, A. Liu, A. Qi, W. Zheng. IEEE Photon. Technol. Lett., 29 (23), 2005 (2017).

[4] Н.А. Берт, А.Д. Бондарев, В.В. Золотарев, Д.А. Кириленко, Я.В. Лубянский, А.В. Лютецкий, С.О. Слипченко, А.Н. Петрунов, Н.А. Пихтин, К.Р. Аюшева, И.Н. Арсентьев, И.С. Тарасов. ФТП, 49 (10), 1429 (2015).

[5] Д.А. Веселов, В.А. Капитонов, Н.А. Пихтин, А.В. Лютецкий, Д.Н. Николаев, С.О. Слипченко, З.Н. Соколова, В.В. Шамахов, И.С. Шашкин, И.С. Тарасов. Квант. электрон., 44 (11), 993 (2014).

[6] J. Piprek. Optical Quant. Electron., 51 (2), 60 (2019).

[7] H. Lamela, B. Roycroft, P. Acedo, R. Santos, G. Carpintero. Optics Lett., 27 (5), 303 (2002).

[8] С.О. Слипченко, А.А. Подоскин, Д.А. Винокуров, А.Л. Станкевич, А.Ю. Лешко, Н.А. Пихтин, В.В. Забродский, И.С. Тарасов. ФТП, 45 (10), 1431 (2011).

[9] S.O. Slipchenko, A.A. Podoskin, N.A. Pikhtin, I.S. Tarasov. Laser Phys., 24 (10), 105001 (2014).

[10] A. Komissarov, M. Maiorov, R. Menna, S. Todorov, J. Connolly, D. Garbuzov, V. Khalfin, A. Tsekoun. In: Technical Digest. Summaries of papers presented at the Conference on Lasers and Electro-Optics. Postconference Technical Digest [IEEE Cat. No.01CH37170] (2001) p. 31.

Редактор А.Н. Смирнов 


\section{Light characteristics of narrow-stripe high-power semiconductor lasers $(1060 \mathrm{~nm})$ based on asymmetric AIGaAs/GaAs heterostructures with a broad waveguide}

I.S. Shashkin, A.Y. Leshko, D.N. Nikolaev, V.V. Shamakhov, N.A. Rudova, K.V. Bakhvalov, A.V. Lutetskiy, V.A. Kapitonov, V.V. Zolotarev, S.O. Slipchenko, N.A. Pikhtin, P.S. Kop'ev

loffe Institute,

194021 St. Petersburg, Russia

Abstract Light characteristics of narrow-stripe lasers $(5.5 \mu \mathrm{m})$ based on asymmetric $\mathrm{AlGaAs} / \mathrm{GaAs}$ heterostructures are studied. It was shown that the maximum optical power achieved under continuous-wave $(\mathrm{CW})$ operation is limited by thermal heating and reaches $1695 \mathrm{~mW}$ at a current of $2350 \mathrm{~mA}$ at $+25^{\circ} \mathrm{C}$, and the maximum efficiency reaches $54.8 \%$. By reducing the operating temperature to $-8^{\circ} \mathrm{C}$, we were able to increase the maximum power to $2 \mathrm{~W}$. A peak power of $2930 \mathrm{~mW}$ was obtained under pulsed operation (pulse width $240 \mathrm{~ns}$, amplitude $4230 \mathrm{~mA}$ ). It is shown there is a region of an „optical dip“ in the power profile with a low-efficiency lasing of a train of pulses of sub-ns duration under pulsed operation. 